\title{
Análisis de las hospitalizaciones por bacteriemia relacionada con el catéter de hemodiálisis
}

\author{
Cristina Carrasco Salinas, Margarita Ruiz García, Victoria Eugenia Gómez López, Rafael Méndez Ordóñez, \\ María López Andreu*, Rodolfo Crespo Montero
}

Enfermera/o, *Nefróloga, Unidad de Gestión Clínica Nefrología, Hospital Universitario Reina Sofía de Córdoba

\section{Resumen}

El uso de catéteres venosos centrales para hemodiálisis se ha incrementado de tal forma, que actualmente en nuestro país, el $30 \%$, de los pacientes son portadores de este acceso vascular. No obstante su duración es limitada y presentan con frecuencia complicaciones, como por ejemplo bacteriemias relacionadas con el catéter de hemodiálisis. Esta, es una complicación con elevada morbimortalidad en los pacientes en tratamiento renal sustitutivo crónico.

El objetivo de este análisis fue conocer la incidencia de hospitalizaciones, identificar los gérmenes más frecuentemente relacionados en nuestra población y analizar los factores demográficos y clínicos asociados.

Se realizó un estudio observacional prospectivo en el que se analizaron todos los ingresos por bacteriemia relacionada con el catéter de hemodiálisis durante el año 2011. Se recogieron los datos clínicos de los pacientes, demográficos y comorbilidad.

El número total de ingresos por bacteriemias relacionadas con el catéter fue de 45 en 35 pacientes, con una edad media de 69.9 años. Los gérmenes más frecuentemente aislados fueron cocos gram positivos (Staphilococcus aureus mecitilin resistente y Staphilococcus aureus meticilin sensible). La mortalidad fue de $14 \%$ (5 pacientes).

\section{Correspondencia: \\ Rodolfo Crespo Montero \\ Unidad Gestión Clínica Nefrología \\ Hosp. Universitario Reina Sofía \\ Avda. Menéndez Pidal s/n. 14005 Córdoba \\ E-mail: rodolfo.crespo.sspa@juntadeandalucia.es}

A la vista de nuestros resultados, podemos concluir que la bacteriemia relacionada con el catéter es una complicación grave en los pacientes en hemodiálisis. Representa casi el $50 \%$ de los ingresos durante 2011 de los pacientes en hemodiálisis. Está asociada con una elevada morbilidad y consumo de recursos.

\section{PALABRAS CLAVE: \\ - CATÉTER VENOSO CENTRAL \\ - HEMODIÁLISIS \\ - BACTERIEMIA \\ - COMPLICACIONES}

\section{Analysis of hospitalizations due to bacteraemia related to the haemodialysis catheter}

\section{Abstract:}

The use of central venous catheters for haemodialysis has increased to such an extent that in our country $30 \%$ of patients now have this vascular access. However their duration is limited and they frequently present complications, such as bacteraemia related to the haemodialysis catheter. This is a complication with high morbimortality in patients undergoing chronic renal replacement therapy.

The aim of this analysis was to determine the incidence of hospitalizations, identify the germs most frequently found in our population and analyse the associated demographic and clinical factors.

A prospective observational study was carried out in which all hospital admissions during 2011 for bacte- 
raemia related to the haemodialysis catheter were analysed. Clinical, demographic and comborbility data of the patients were compiled.

The total number of admissions for catheter-related bacteraemia was 45 in 35 patients, with a mean age of 69.9 years. The most frequently isolated germs were gram-positive cocci (Methicillin-Resistant Staphylococcus Aureus and Methicillin-Sensitive Staphylococcus Aureus). Mortality was 14\% (5 patients).

In light of our results, we may conclude that catheter-related bacteraemia is a serious complication in haemodialysis patients. It represents almost $50 \%$ of hospital admissions in 2011 of haemodialysis patients. It is associated to high morbility and consumption of resources.

\section{KEY WORDS:}

- CENTRAL VENOUS CATHETER

- HAEMODIALYSIS

- BACTERAEMIA

- COMPLICATIONS

\section{Introducción}

La fístula arteriovenosa (FAV) autóloga es el acceso vascular para hemodiálisis (HD) con mayor supervivencia, menor número de infecciones y menor coste ${ }^{1-3}$. Sin embargo, el aumento de edad del paciente y la comorbilidad asociada determina que cada vez, un mayor número de pacientes se dialicen a través de catéter venoso central (CVC) ${ }^{4,5}$.

Los CVC se asocian con un mayor número de disfunciones, infecciones y hospitalizaciones ${ }^{6,7}$. Una de las complicaciones infecciosas que origina aumento de las hospitalizaciones es la bacteriemia relacionada con el catéter (BRC). La BRC es una de las principales causas de morbilidad, hospitalizaciones y mortalidad en los pacientes en tratamiento renal sustitutivo mediante $\mathrm{HD}^{8,9}$. Se ha asociado a la BRC, como principales complicaciones, endocarditis infecciosa, artritis séptica, embolismo séptico, osteomielitis y espondilodiscitis, absceso epidural y sepsis severa ${ }^{10,11}$.

Aunque la etiología de las mismas puede variar, el germen más frecuentemente aislado en los pacientes con
BRC es el Staphilococus Aureus, siendo igualmente el germen implicado en las complicaciones más severas como resultado de la predilección del S. Aureus por las válvulas cardiacas y tejido óse ${ }^{12}$.

Las altas tasas de complicaciones asociadas con la bacteriemia relacionada con el CVC de hemodialisis (BRC) implican una elevada tasa de hospitalización y el aumento de los costes para el sistema sanitario con un mayor consumo de recursos ${ }^{13}$.

El objetivo de presente estudio fue, por tanto, realizar un análisis retrospectivo de las BRC graves que precisaron hospitalización en la Unidad de Gestión Clínica de Nefrología en el año 2011, así como la etiología bacteriana de nuestra muestra.

\section{Material y métodos}

Se ha realizado un estudio descriptivo y retrospectivo en el Hospital Reina Sofía de Córdoba. Se trata de un hospital universitario de tercer nivel con un total de 1233 camas con una población de referencia de 1.144.934 personas. La población de referencia provincial para la Unidad de Nefrología es de 790.584 personas.

Se incluyeron todos los pacientes con insuficiencia renal crónica en tratamiento con HD que precisaron hospitalización en la Unidad de Gestión Clínica de Nefrología del Hospital Universitario Reina Sofía, desde Enero a Diciembre de 2011.

Del total de hospitalizaciones en el año, 125 episodios, se seleccionaron, según los datos de CMBD del año 2011, a los pacientes con diagnóstico de Sepsis con complicación mayor (GRD 584), obteniéndose un total 45 hospitalizaciones en 35 pacientes. No se tuvieron en cuenta, los pacientes con BRC que recibieron tratamiento antibiótico intravenoso, administrado durante la realización de la sesión de HD y que no precisaron hospitalización.

La etiología de la insuficiencia renal se muestra en la tabla $n^{0} 1$.

Se incluyeron como variables de estudio:

- Días de hospitalización, cambio de catéter, germen aislado, características clínicas y demográficas de los pacientes incluidos. 
Para el análisis estadístico se utilizó la distribución de frecuencias para las variables cualitativas y la media \pm desviación típica para variables numéricas. Se utilizó la prueba de Chi-cuadrado y $\mathrm{t}$ de Student para las comparaciones entre grupos. El análisis se realizó con el programa informático SPSS versión 15.0. Se aceptó significación estadística para $p<0.05$.

\begin{tabular}{|l|c|}
\hline Etiología Insuficiencia Renal Crónica & $\mathbf{N}^{0}$ pacientes \\
\hline Desconocida & 14 \\
\hline Pielonefritis/nefritis intersticial & $\mathbf{3}$ \\
\hline Nefroangioesclerosis & $\mathbf{1}$ \\
\hline Nefropatía diabética & $\mathbf{5}$ \\
\hline Poliquistosis & $\mathbf{3}$ \\
\hline Glomerulonefritis extracapilar & $\mathbf{4}$ \\
\hline Amiloidosis & $\mathbf{2}$ \\
\hline Mieloma Múltiple & $\mathbf{1}$ \\
\hline Toxicidad farmacológica & $\mathbf{1}$ \\
\hline Neoplasia & $\mathbf{1}$ \\
\hline
\end{tabular}

Tabla 1.

\section{Resultados}

La edad media de los pacientes incluidos en el estudio fue de $69,91 \pm 14.96$ años, siendo 24 pacientes mayores de 70 años $(68,6 \%$ población). El 51,4\% de los pacientes fueron hombres (18) y el $48.6 \%$ mujeres (17). EI $88.6 \%$ de los pacientes presentaban antecedentes de HTA, el $28.6 \%$ obesidad y el $28.6 \%$ fueron diabéticos.

Un total de 5 pacientes tuvieron más de un ingreso por bacteriemia a lo largo de 2011: un paciente presentó 4 ingresos, dos pacientes 3 ingresos y dos pacientes con 2 ingresos, con diagnostico de sepsis secundaria a bacteriemia relacionada con el acceso vascular.

Del total de pacientes ingresados por BRC, todos eran portadores de un CVC: 34 pacientes con CVC tunelizado y 1 paciente con CVC temporal. No se produjo ningún ingreso por bacteriemia en pacientes con injerto vascular o con FAV funcionante.

De los 35 pacientes, 28 eran portadores de FAV previa no funcionante o trombosada y 3 pacientes eran portadores de catéter por periodo de maduración de la FAV ya realizada; otro paciente estaba en aprendizaje de diálisis peritoneal continua ambulatoria. Solo en 3 pacientes, no se había realizado aún una $\mathrm{FAV}$, que se realizó posteriormente, debido a ser pacientes agudos sin seguimiento previo por nefrología.
Los pacientes estuvieron ingresados una media de 10.5 días, durante el episodio de BRC y se registraron cambio de catéter en 21 ingresos. Todos los pacientes recibieron tratamiento antibiótico intravenoso durante la hospitalización. En el 52,5\% de las hospitalizaciones por sepsis secundaria a BRC fue necesario el tratamiento con aminas vasoactivas, por hipotensión refractaria en algún momento del ingreso.

Los gérmenes más frecuentemente aislados fueron: gram positivos $(63,4 \%$ de los episodios de bacteriemia), siendo el Staphilococcus Aureus meticilin resistente (SAMR) y el Staphilococcus epidermidis meticilin resistente, los gérmenes que mayor número de ingresos por bacteriemia provocaron, 12 y 12 respectivamente (24 en total). Sólo en 2 episodios no se identificó el germen causante de la bacteriemia en los hemocultivos extraídos. La etiología de las bacteriemias se muestra en la tabla $\mathbf{n}^{0} \mathbf{2}$.

\begin{tabular}{|l|c|}
\hline Bacteria aislada & $\mathbf{N}^{0}$ bacteriemias \\
\hline SAMR & 12 \\
\hline SAMS & $\mathbf{7}$ \\
\hline Stafilococo epidermidis Meticilin Resistente & $\mathbf{1 2}$ \\
\hline Pseudomona aeruginosa & $\mathbf{3}$ \\
\hline Enterobacter cloacae & $\mathbf{5}$ \\
\hline E coli & $\mathbf{2}$ \\
\hline Polimicrobiana & $\mathbf{1}$ \\
\hline No germen & $\mathbf{2}$ \\
\hline
\end{tabular}

Tabla 2.

La mortalidad fue del 14,28\% (fallecieron 5 pacientes hospitalizados por BRC).

Cuando separamos a los pacientes en función de que el germen causante de bacteriemia fuera meticilin resistente o no, y se comparó la estancia media, el cambio de catéter, el reingreso y el número de éxitus, se observó que la estancia media fue significativamente mayor en los pacientes con BRC producida por germen meticilin resistentes (SAMR y S. epidermidis, $p<0,009$ ).

\section{Discusión}

La BRC y los episodios de sepsis secundaria son una de las complicaciones con mayor morbimortalidad en los pacientes con enfermedad renal terminal en tratamiento con HD. La frecuencia de BRC comunicada es variable y oscila entre 0,74 y 9,15 eventos/1.000 días- 
catéter $^{14,15}$. En efecto, las infecciones son la segunda causa de mortalidad en los pacientes con enfermedad renal crónica terminal, por detrás de los eventos cardiovasculares. EI $75 \%$ de la mortalidad asociada a las infecciones es secundaria a un episodio de sepsis ${ }^{8}$.

En el estudio HEMO se muestra un incremento significativo en la mortalidad relacionada con infecciones en pacientes en HD a través de CVC frente a pacientes portadores de FAV (RR 2.30, 95\% CI 1.45-3.64) ${ }^{16}$. Otro estudio multicentrico, prospectivo realizado en Estado Unidos con un total de 1041 pacientes, mostró que el uso de CVC para HD estaba asociado con un aumento del riesgo de mortalidad del $50 \%$, y con un incremento de riesgo de muerte relacionada con una infección del $41 \%$, comparado con los pacientes que eran portadores de FAV como acceso para HD ${ }^{17}$.

En nuestra población de estudio el $84,1 \%$ de los pacientes eran portadores de FAV trombosada o no funcionante, previa a la colocación de CVC. Se trata de una población con elevada dificultad o imposibilidad para obtener un nuevo acceso vascular tipo FAV o injerto vascular, con menor tasas de complicaciones infecciosas. La tasa de mortalidad asociada a la hospitalización por sepsis secundaria a BRC durante el año 2011 fue del 14,28\% (5 pacientes), menor que la reportada en la literatura a pesar de las características de nuestra población ${ }^{8,9}$.

Con respecto a las hospitalizaciones en la Unidad por BRC y sepsis relacionada con el CVC de HD y el germen causante, encontramos una mayor incidencia por gram positivos, siendo el germen más frecuentemente aislado el SAMR, seguido del $\mathrm{S}$ epidermidis meticilin resistente, en la línea de lo publicado por otros autores ${ }^{12}$. La elevada tasa de incidencia en nuestra serie, de gérmenes meticilin resistentes, probablemente este en relación con la población estudiada, pues fueron pacientes que precisaron hospitalización, por cuadro séptico asociado a la bacteriemia. Los pacientes con episodios de sepsis secundaria a BRC por gérmenes meticilin resistentes, mostraron un incremento significativo, de los días de hospitalización, así como un mayor número de recambios de CVC.

Además, hay que indicar que los pacientes que precisaron hospitalización son pacientes con elevada prevalencia de hipertensión arterial, diabetes mellitus, y aunque, con una edad media de 69 años, el $68 \%$ de los mismos estaba por encima de los 70 años de edad. Las características de este grupo de pacientes, podría explicar el desarrollo de sepsis secundaria a la BRC, y la necesidad de recambio de CVC.
Por otra parte, es importante resaltar que la hospitalización por BRC supone un incremento en el consumo de recursos de los pacientes en tratamiento con $\mathrm{HD}^{18}$. A esto hay que añadir que dializarse a través de un CVC supone un coste medio mensual, adicional al tratamiento de la HD de $561{ }^{19}$.

Pese a las recomendaciones, y debido a las características de la población incidente en HD, cada vez es más frecuente en determinados estratos de esta población (mayores de 75 años, con elevada tasa de patología vascular y obesidad) la presencia de CVC como acceso vascular. Por tanto, la mejor estrategia para la disminución del número de episodios de BRC y por consiguiente, para la reducción de morbimortalidad en los pacientes y reducción de costes asociados, es la prevención. Varios estudios, hacen especial hincapié en la necesidad de cumplir los procedimientos de manejo de los CVC, con máxima rigurosidad y asepsia, como medida eficaz para la prevención de la $\mathrm{BRC}^{12}$, y que en esta tarea la concienciación y labor de enfermería es clave ${ }^{20}$.

Por todo ello, es necesario continuar realizando estudios individualizados de los pacientes para lograr un acceso vascular tipo FAV o injerto para HD, medidas encaminadas a la detección precoz de la infección relacionada con el catéter y asepsia exhaustiva en el proceso de conexión y desconexión de los pacientes; para lograr disminuir las tasas de bacteriemia y hospitalización secundaria, los costes asociados, así como la morbimortalidad de estos pacientes.

A la vista de nuestros resultados podemos concluir que las sepsis secundaria a BRC en los pacientes portadores de CVC cuya infección ha sido provocada por gérmenes meticilin resistentes, presentan mayor número de días de ingreso y necesidad de de recambio de catéter.

\section{Bibliografía}

1. NKF-K/DOQI (4): Clinical practice guidelines for vascular access: update 2000. Am J Kidney Dis 2001; 37(1): S137-81.

2. Konner K, Hulbert-Shearon TE, Roys EC, Port FK. Tailoring the initial vascular access for dialysis patients. Kidney Int 2002; 62(1): 329-338.

3. Manns $P$, Tonelli M, Yilmaz $S$, Lee $H$, Laupland $\mathrm{K}$, Klarenbach $S$, et al. Establishment and maintenance of vascular access in incident hemodialysis 
patients: a prospective cost analysis. J Am Soc Nephrol 2005; 16(1): 201-209.

4. Pisoni RL, Young EW, Dykstra DM, Greenwood RN, Hecking $E$, Gillespie $B$, et al. Vascular access use in Europe and in the United States: Results from the DOPPS. Kidney Int 2002; 61(1): 305-316.

5. Ethier J, Mendelssohn DC, Elder SJ, Akiba T, et al. Vascular access use and outcomes: an international perspective from the dialysis outcomes and practice patterns study. Nephrol. Dial Trasplant 2008; 23(10): 3219-3226.

6. Nassar GM, Ayus JC. Infectious complications of the hemodialysis access. Kidney Int 2001; 60(1): 1-13.

7. Rayner HC, Pisoni RL, Bommer J, Canaud B, Hecking $E$, Locatelli $F$, et al. Mortality and hospitalization in haemodialysis patients in five European countries: Results from the Dialysis Outcomes and Practice Patterns Study (DOPPS). Nephrol Dial Transplant 2004; 19(1): 108-120.

8. Jaber BL. Bacterial infections in hemodialysis patients: pathogenesis and prevention. Kidney Int 2005; 67(6): 2508-2519.

9. Dopirak M et al. Surveillance of hemodialysis associated primary bloodstream infections: the experience of ten hospital-based centers. Infect Control Hosp Epidemiol 2002; 23(12): 721-724.

10. Krishnasami $Z$, et al. Management of hemodialysis catheter-related bacteremia with an adjunctive antibiotic lock solution. Kidney Int 2002; 61: 11361142

11. Tanriover B, et al. Bacteremia associated with tunneled dialysis catheters: comparison of two treatment strategies. Kidney Int 2000; 57: 2151-2155

12. Albalate $M$, Pérez Garcíal $R$, De Sequera $P$, y cols. ¿Hemos olvidado lo más importante para prevenir las bacteriemias en pacientes portadores de catéteres para hemodiálisis? Nefrología 2010; 30(5): 573-577.

13. $\mathrm{Ng} \mathrm{Lj}$, Chen F, Pisoni RL et al. Hospitalization risks related to vascular access type among incident US hemodialysis patients. Nephrol Dial Transplant 2011; 0:1-8

14. Power A, Duncan N, Singh SK, et al. Sodium citrate versus heparin catheter locks for cuffed central venous catheters: a single-center randomized controlled trial. Am J Kidne<y Dis 2009; 53(6): 1034-1041.

15. Feely T, Copley A, Bleyer AJ. Catheter lock solutions to prevent bloodstream infections in high-risk hemodialysis patients. Am J Nephrol 2007;27: 2429

16. Allon M, Depner $T$, Radeva M, et al. Impact of dialysis dose and membrane on infection-related hospitalization and death: results of the HEMO Study. J Am Soc Nephrol 2003; 14(7): 1863-1870.

17. Astor BC, Eustace J, Powe N, et al. Type of vascular access and survival among incident hemodialysis patients: the Choices for Healthy Outcomes in Caring for ESRD (CHOICE) Study. J Am Soc Nephrol 2005; 16(5): 1449-1455

18. Parra $E$, Arenas MD, Alonso $M$, y cols. Estudio multicéntrico de costes en hemodiálisis. Nefrología 2011; 31(3): 299-307.

19. Gruss E, Portolés J, Tato A, Hernández T, y cols. Repercusiones clínicas y económicas del uso de catéteres tunelizados de hemodiálisis en un área sanitaria. Nefrología 2009; 29(2): 123-129.

20. Martín Chacón E, Martín Álvarez MC, Sánchez Calzado $\mathrm{M}$, y cols. Incidencia de bacteriemia en pacientes portadores de catéter permanente tunelizado para hemodiálisis. Rev Soc Esp Enferm Nefrol 2008; 11(4): 277-281. 